\title{
ASYMPTOTICALLY OPTIMAL ERROR BOUNDS FOR QUADRATURE RULES OF GIVEN DEGREE
}

\author{
H. BRASS
}

\begin{abstract}
If the quadrature rule $Q$ is applied to the function $f$, then the error can in many situations be bounded by $\rho_{s}(Q)\left\|f^{(s)}\right\|_{\infty}$, where $\rho_{s}(Q)$ is independent of $f$. We obtain the asymptotics of these numbers for the Gaussian method $Q_{n}^{\mathbf{G}} \quad(n=1,2, \ldots)$ with very general weight functions and show that $\rho_{s}\left(Q_{n}^{\mathrm{G}}\right)$ is (asymptotically) an upper bound for $\rho_{s}(Q)$, if $Q$ is any quadrature rule with the same degree as $Q_{n}^{\mathrm{G}}$.
\end{abstract}

\section{INTRODUCTION}

Let $I$ denote a fixed given functional on $C[-1,1]$ of the form

$$
I[f]:=\int_{-1}^{1} f(x) k(x) d x \quad \text { with } k \in L[-1,1], k \geq 0, \int_{-1}^{1} k(x) d x>0 .
$$

The numerical computation of $I[f]$ is often done with the help of "quadrature rules" $Q$; these are functionals of type

$$
Q[f]:=\sum_{\nu=1}^{n} a_{\nu} f\left(x_{\nu}\right) \quad \text { with } a_{\nu} \geq 0,-1 \leq x_{1}<\cdots<x_{n} \leq 1 .
$$

The error is the functional $R:=I-Q$. The degree of $Q$ is the number $\operatorname{deg}[Q]:=\sup \left\{m: R\left[\mathscr{P}_{m}\right]=0\right\}$, where $\mathscr{P}_{m}$ denotes the space of polynomials of degree $\leq m$. The most interesting quadrature rules are the Gaussian rules $Q_{n}^{\mathrm{G}}$, which are characterized as rules with $n$ evaluation points and degree $2 n-1$.

We are interested in error bounds of the type

$$
|R[f]| \leq \rho_{s}(Q)\left\|f^{(s)}\right\|
$$

$(\|\cdot\|$ means the sup norm throughout this paper), that is, in the numbers

$$
\rho_{s}(Q):=\sup \left\{|R[f]|: f \in \mathscr{K}_{s}\right\}, \quad \mathscr{K}_{s}:=\left\{f:\left\|f^{(s)}\right\| \leq 1\right\} .
$$

We shall determine the asymptotics of $\rho_{s}\left(Q_{n}^{\mathrm{G}}\right)$ and shall prove that these numbers are (asymptotically) upper bounds for $\rho_{s}(Q)$, if $\operatorname{deg}[Q]=\operatorname{deg}\left[Q_{n}^{\mathrm{G}}\right]$.

As an illustration we cite a special result: Let $k=1$ and let $Q_{1}, Q_{2}, \ldots$ be a sequence of quadrature rules with $\operatorname{deg}\left[Q_{m}\right] \geq m-1$. Then we have

$$
\lim _{m \rightarrow \infty} m \rho_{1}\left(Q_{m}\right) \leq \frac{\pi^{2}}{4}=\lim _{m \rightarrow \infty} m \rho_{1}\left(Q_{\lfloor(m+1) / 2\rfloor}^{\mathrm{G}}\right) .
$$

Received by the editor April 2, 1992.

1991 Mathematics Subject Classification. Primary 41A55. 
If we make the further assumption that $Q_{m}$ has $m$ evaluation points, then we have $m \rho_{1}\left(Q_{m}\right) \geq 1$ (see, e.g., [1, p. 242]). Hence, all quadrature rules of interpolatory type (Gauss, Lobatto, Radau, Clenshaw/Curtis, Filippi, ... ) are of similar quality with respect to $\rho_{1}$, and there is only little improvement possible if we would use the best rule (which is not of interpolatory type and will give poor results if applied to functions of high smoothness).

To be more precise, we define

$$
\rho_{s, m}:=\sup \left\{\rho_{s}(Q): \operatorname{deg}[Q] \geq m\right\}, \quad 1 \leq s \leq m+1 .
$$

With suitable assumptions on $k$ we shall prove

$$
\lim _{m \rightarrow \infty} m^{s} \rho_{s, m}=\lim _{m \rightarrow \infty} m^{s} \rho_{s}\left(Q_{\lceil(m+1) / 2\rceil}^{\mathrm{G}}\right)=(2 \pi)^{s} I\left[h^{s}\right] \int_{0}^{1}\left|B_{s}(x)\right| d x,
$$

where we have used the notation

$$
h(x):=\sqrt{1-x^{2}}, \quad B_{s}(x):=-2 \sum_{\mu=1}^{\infty} \frac{\cos \left(2 \pi \mu x-\frac{1}{2} \pi s\right)}{(2 \pi \mu)^{s}} .
$$

The restriction of $B_{s}$ to $(0,1)$ is identical with the Bernoulli polynomial (see, e.g., Hämmerlin and Hoffman [8]).

Formula (1.1) gives the asymptotics of $\rho_{s, m}$ if $s$ is fixed, but there are many error bounds which use an increasing order of derivatives. For this situation we shall prove: If $l \in\{0,1, \ldots\}$ is fixed, then

$$
\begin{aligned}
\lim _{m \rightarrow \infty} \frac{2^{m+1}(m+1) !}{m^{l / 2}} \rho_{m-l+1, m} & =\lim _{m \rightarrow \infty} \frac{2^{m+1}(m+1) !}{m^{l / 2}} \rho_{m-l+1}\left(Q_{\lceil(m+1) / 2\rceil}^{\mathrm{G}}\right) \\
& =c_{l} \sqrt{\pi} \exp \left(\frac{1}{\pi} \int_{-1}^{1} \frac{\ln k(x)}{\sqrt{1-x^{2}}} d x\right)
\end{aligned}
$$

with

$$
c_{l}:=\int_{-\infty}^{\infty}\left|D^{l}\left[e^{-x^{2}}\right]\right| d x, \quad D[f]:=f^{\prime} .
$$

\section{THE RESULTS}

Theorem 1. Let $Q_{1}, Q_{2}, \ldots$ be a sequence of quadrature rules with $\operatorname{deg}\left[Q_{m}\right] \geq$ $m$; then we have

$$
\varlimsup_{m \rightarrow \infty} m^{s} \rho_{s}\left(Q_{m}\right) \leq(2 \pi)^{s} I\left[h^{s}\right] \int_{0}^{1}\left|B_{s}(x)\right| d x, \quad h(x)=\sqrt{1-x^{2}} .
$$

In the special case $s=1$, this theorem is given in [4]; under the further assumption $k=1$ an asymptotically sharp bound is given in the same paper.

The constants

$$
K_{s}:=\frac{4}{\pi} \sum_{\nu=0}^{\infty} \frac{(-1)^{\nu(s+1)}}{(2 \nu+1)^{s+1}}, \quad s=0,1, \ldots,
$$

are called Favard constants; they are used in our next theorem. 
Theorem 2. Suppose $v$ is continuous on $(-1,1)$ and $M:=\inf \{v(x):-1<$ $x<1\}>0$ holds. Let $Q_{1}, Q_{2}, \ldots$ be a sequence of quadrature rules

$$
Q_{n}[f]:=\sum_{\nu=1}^{n} a_{\nu, n} f\left(x_{\nu, n}\right)
$$

with

$$
\lim _{n \rightarrow \infty} n^{-1}\left|\left\{x_{\nu, n}: x_{\nu, n} \leq t\right\}\right|=\int_{-1}^{t} v(x) d x
$$

$(|\{\cdots\}|$ means the cardinality of $\{\cdots\})$; then we have

$$
\varliminf_{n \rightarrow \infty} n^{s} \rho_{s}\left(Q_{n}\right) \geq(2 \pi)^{-s} K_{s} I\left[v^{-s}\right] .
$$

The condition (2.1) is satisfied for the Gaussian method $Q_{n}^{\mathrm{G}}(n=1,2, \ldots)$ with $v(x):=\pi^{-1}\left(1-x^{2}\right)^{-1 / 2}$ (and for the Lobatto method and the Radau methods) if $k>0$ a.e. (see, e.g., Freud [7, Theorem 9.2]).

If $s$ is odd, then we have

$$
K_{s}=(2 \pi)^{s} \int_{0}^{1}\left|B_{s}(x)\right| d x ;
$$

for the proof, use $B_{s}(x) \neq 0$ if $x \in\left(0, \frac{1}{2}\right)$, see [8]. With the aid of (2.2) we obtain by a combination of Theorem 1 and Theorem 2 as a main result of this paper:

Theorem 3. Let $s$ be odd, and suppose $k>0$ a.e. Then (1.1) holds.

$$
K_{s} I\left[h^{s}\right] \leq \varliminf_{m \rightarrow \infty} m^{s} \rho_{s, m} \leq \varlimsup_{m \rightarrow \infty} m^{s} \rho_{s, m} \leq(2 \pi)^{s} I\left[h^{s}\right] \int_{0}^{1}\left|B_{s}(x)\right| d x
$$

if $k>0$ a.e. However, the ratio of the bounds is $<1.027$.

If we make stronger assumptions on $k$, we can prove (1.1) also for even $s$. We have to use the following theorem of Petras [10]:

Theorem. If there is a constant $M$ and a polynomial $\tau$ with zero set $N:=$ $\left\{-1, \xi_{1}, \ldots, \xi_{m}, 1\right\}$ such that

$$
\tau^{2}(x) \leq k(x) \leq M\left(1-x^{2}\right)^{-1 / 2}, \quad x \in[-1,1] \backslash N,
$$

and if $k$ is continuous on $[-1,1] \backslash N$, then we have

$$
\lim _{n \rightarrow \infty} n^{s} \rho_{s}\left(Q_{n}^{\mathrm{G}}\right)=\pi^{s} I\left[h^{s}\right] \int_{0}^{1}\left|B_{s}(x)\right| d x .
$$

As an immediate consequence we have

Theorem 4. The relation (1.1) holds under the conditions of the theorem of Petras.

We turn now to the case of increasing $s$. Here we have 
Theorem 5. If $\int_{-1}^{1}\left(1-x^{2}\right)^{-1 / 2} \ln k(x) d x$ exists, then (1.3) holds.

With the added hypothesis $k=1$, the exact values of $\rho_{m+1, m}$ are obtained in [6], [3]; with the same hypothesis, the asymptotics of $\rho_{m-l+1}\left(Q_{\lceil(m+1) / 2\rceil}^{\mathrm{G}}\right)$ is given in [5].

The proof of Theorem 1 uses two results on the degree of approximation by (ordinary or trigonometric) polynomials, which are of independent interest. We use the notation

$$
\hat{a}_{\nu}[f]:=\frac{2}{\pi} \int_{-1}^{1} f(x) T_{\nu}(x)\left(1-x^{2}\right)^{-1 / 2} d x \quad \text { with } T_{\nu}(x):=\cos (\nu \arccos x)
$$

and state

Theorem 6. If $f \in \mathscr{K}_{s}$, then there exists a sequence $p_{0}, p_{1}, \ldots$ with

(i) $p_{n} \in \mathscr{P}_{n}$,

(ii) $\left|f(x)-p_{n}(x)\right| \leq(2 \pi)^{s}(n+1)^{-s}\left(\sqrt{1-x^{2}}\right)^{s} \int_{0}^{1}\left|B_{s}(u)\right| d u+O\left(n^{-s-1}\right)$,

(iii) $\hat{a}_{\nu}[f]-\hat{a}_{\nu}\left[p_{n}\right]=O\left(n^{-s-1}\right), \nu=0,1, \ldots$.

The $O$ terms hold uniformly in $\mathscr{K}_{s}$.

We shall need some further notations:

$$
\begin{gathered}
\mathscr{T}_{n}:=\left\{t: t(x)=\sum_{\nu=0}^{n}\left(\alpha_{\nu} \cos \nu x+\beta_{\nu} \sin \nu x\right) ; \alpha_{\nu}, \beta_{\nu} \in \mathbb{R}\right\}, \\
a_{\nu}[f]:=\frac{1}{\pi} \int_{-\pi}^{\pi} f(x) \cos (\nu x) d x, b_{\nu}[f]:=\frac{1}{\pi} \int_{-\pi}^{\pi} f(x) \sin (\nu x) d x .
\end{gathered}
$$

We can now state

Theorem 7. Let $f$ be a $2 \pi$-periodic function with a bounded sth derivative. Then there exists a sequence $t_{0}, t_{1}, \ldots$ with

(i) $t_{n} \in \mathscr{T}_{n}$,

(ii)

$$
\left\|f-t_{n}\right\| \leq \frac{(2 \pi)^{s}}{(n+1)^{s}} \int_{0}^{1}\left|B_{s}(u)\right| d u \inf \left\{\left\|f^{(s)}-c\right\|: c \in \mathbb{R}\right\}
$$

$$
\begin{aligned}
& \left|a_{\nu}\left[f-t_{n}\right]\right|=O\left(n^{-s-1}\right) \inf \left\{\left\|f^{(s)}-c\right\|: c \in \mathbb{R}\right\}, \\
& \left|b_{\nu}\left[f-t_{n}\right]\right|=O\left(n^{-s-1}\right) \inf \left\{\left\|f^{(s)}-c\right\|: c \in \mathbb{R}\right\} .
\end{aligned}
$$

If $s$ is odd, then (i), (ii) is the well-known theorem of Favard, Achieser and Krein (see, e.g., Timan [12, p. 289]) and the method of proof leads to (iii) too. Therefore, we shall restrict ourselves to the proof of Theorem 7 in the special case of even $s$.

\section{Proof of Theorem 7}

Lemma 1. Let $s \in \mathbb{N}$ be fixed. There exists a sequence $u_{n}\left(=u_{n, s}\right) \quad(n=$ $1,2, \ldots)$ such that

(i) $u_{n} \in \mathscr{T}_{n}$,

(ii) $a_{0}\left(u_{n}\right)=0$, 
(iii)

$$
\begin{aligned}
& a_{\lambda}\left[B_{s}\left(\frac{\dot{ }}{2 \pi}\right)-u_{n}\right]=O\left(n^{-s-1}\right), \\
& b_{\lambda}\left[B_{s}\left(\frac{\dot{2}}{2 \pi}\right)-u_{n}\right]=O\left(n^{-s-1}\right), \quad \lambda=1,2, \ldots,
\end{aligned}
$$

(iv) The function $B_{s}(\dot{\dot{\pi} \pi})-u_{n}$ changes its sign on $(0,2 \pi)$ exactly at the zeros of $B_{s}((n+1) \dot{\overline{2}})$.

Proof. Let $s$ be even. (The lemma holds for all $s \in \mathbb{N}$, but for our purpose we need it only for $s$ even.) The function $B_{s}$ has two zeros $\xi_{1} \in\left(0, \frac{1}{2}\right)$ and $\xi_{2}=1-\xi_{1}$ on $[0,1]\left(\left[8\right.\right.$, p. 280]). We define the functions $u_{n}$ of the lemma by

$$
\begin{aligned}
u_{n}(x) & :=\sum_{\lambda=1}^{n} \alpha_{\lambda, n} \cos \lambda x, \\
\alpha_{\lambda, n} & :=\frac{2(-1)^{(s+2) / 2}}{(2 \pi)^{s} \sin \left(2 \pi \xi_{1}\right)} \sum_{\nu=-\infty}^{\infty} \frac{\sin \left((\nu+1) 2 \pi \xi_{1}\right)}{(\nu(n+1)+\lambda)^{s}} .
\end{aligned}
$$

Then (i) and (ii) are true. After some work we get from the above definitions

$$
\begin{aligned}
& a_{\lambda}\left[B_{s}\left(\frac{\dot{2}}{2 \pi}\right)-u_{n}\right] \\
&=\frac{2(-1)^{s / 2}}{(2 \pi)^{s}}\left(\sum_{\nu=1}^{\infty} \frac{2 \cos \left(\nu 2 \pi \xi_{1}\right)}{(\nu(n+1))^{s}}+\frac{1}{\sin \left(2 \pi \xi_{1}\right)}\right. \\
& \quad \times\left(\sum_{\nu=1}^{\infty} \sin \left((\nu+1) 2 \pi \xi_{1}\right)\left((\nu(n+1)+\lambda)^{-s}-(\nu(n+1))^{-s}\right)\right. \\
&\left.\left.\quad-\sum_{\nu=1}^{\infty} \sin \left((\nu-1) 2 \pi \xi_{1}\right)\left((\nu(n+1)-\lambda)^{-s}-(\nu(n+1))^{-s}\right)\right)\right) .
\end{aligned}
$$

The first sum is $(-1)^{(s+2) / 2}(2 \pi)^{s}(n+1)^{-s} B_{s}\left(\xi_{1}\right)=0$; the second sum can be bounded by

$$
\begin{aligned}
\sum_{\nu=1}^{\infty}\left|(\nu(n+1)+\lambda)^{-s}-(\nu(n+1))^{-s}\right| \\
=(n+1)^{-s} \sum_{\nu=1}^{\infty} \nu^{-s}\left(1-\left(1+\frac{\lambda}{\nu(n+1)}\right)^{-s}\right) \\
\leq(n+1)^{-s} \sum_{\nu=1}^{\infty} \nu^{-s} \frac{\lambda}{\nu(n+1)}=O\left((n+1)^{-s-1}\right) ;
\end{aligned}
$$

for the third sum we use the same method, and (iii) is proven.

For the proof of (iv) we use the notation

$$
x_{\mu, i}:=\left(\mu+\xi_{i}\right) \frac{2 \pi}{n+1}, \quad \mu=0,1, \ldots, n ; i=1,2 .
$$

These are the zeros of $B_{s}\left((n+1) \dot{\frac{\dot{2}}{2 \pi}}\right)$ on $(0,2 \pi)$. Verifying

$$
B_{s}\left(\frac{x_{\mu, i}}{2 \pi}\right)=u_{n}\left(x_{\mu, i}\right), \quad \mu=0,1, \ldots, n ; i=1,2,
$$

is a rather long but elementary task. 
If $d:=B_{s}\left(\frac{\dot{2}}{2 \pi}\right)-u_{n}$ had zeros different from $x_{\mu, i}$, or if one of these zeros would not be a point of sign change, then $d$ would have at least $2 n+3$ zeros on $(0,2 \pi)$. But then $d^{\prime}, d^{\prime \prime}, \ldots, d^{(s-2)}$ would also have at least $2 n+3$ zeros on $[0,2 \pi)$ (Rolle and periodicity), whereas $d^{(s-1)}$ would have $2 n+2$ and $d^{(s)}$ would have $2 n+1$ zeros. But this is a contradiction because of $0 \neq d^{(s)} \in \mathscr{T}_{n}$. Thus we have (iv).

Proof of Theorem 7. Using the $u_{n}$ from Lemma 1, we define

$$
\begin{aligned}
t_{n}(x) & :=\frac{1}{2} a_{0}[f]-(2 \pi)^{s-1} \int_{0}^{2 \pi} u_{n}(x-y) f^{(s)}(y) d y \\
& =\frac{1}{2} a_{0}[f]+(-1)^{(s+2) / 2} 2^{s-1} \pi^{s} \sum_{\lambda=1}^{n} \alpha_{\lambda, n} \lambda^{s}\left(a_{\lambda}[f] \cos (\lambda x)+b_{\lambda}[f] \sin (\lambda x)\right) .
\end{aligned}
$$

Then (i) of Theorem 7 is evidently true, and (iii) follows easily from assertion (iii) of Lemma 1.

If $f$ is $2 \pi$-periodic and has an sth derivative, then we have

$$
f(x)=\frac{1}{2} a_{0}[f]-(2 \pi)^{s-1} \int_{0}^{2 \pi} B_{s}\left(\frac{x-y}{2 \pi}\right) f^{(s)}(y) d y ;
$$

this is a special case of the Euler-Maclaurin sum formula. Combining (3.1) and (3.2), we get

$$
\begin{aligned}
f(x)-t_{n}(x) & =-(2 \pi)^{s-1} \int_{0}^{2 \pi}\left(B_{s}\left(\frac{x-y}{2 \pi}\right)-u_{n}(x-y)\right) f^{(s)}(y) d y \\
& =-(2 \pi)^{s-1} \int_{0}^{2 \pi}\left(B_{s}\left(\frac{z}{2 \pi}\right)-u_{n}(z)\right) f^{(s)}(x-z) d z .
\end{aligned}
$$

The first factor of the integrand has mean value zero; therefore, we can deduce

$$
\left\|f-t_{n}\right\| \leq(2 \pi)^{s-1} \int_{0}^{2 \pi}\left|B_{s}\left(\frac{z}{2 \pi}\right)-u_{n}(z)\right| d z\left\|f^{(s)}-c\right\|,
$$

where $c$ is any constant.

We use now assertion (iv) of Lemma 1:

$$
\int_{0}^{2 \pi}\left|B_{s}\left(\frac{z}{2 \pi}\right)-u_{n}(z)\right| d z=\left|\int_{0}^{2 \pi}\left(B_{s}\left(\frac{z}{2 \pi}\right)-u_{n}(z)\right) \operatorname{sgn} B_{s}\left((n+1) \frac{z}{2 \pi}\right) d z\right| \text {. }
$$

The second factor of the integrand has period $h:=\frac{2 \pi}{n+1}$; hence we obtain

$$
\begin{aligned}
\int_{0}^{2 \pi} & \left|B_{s}\left(\frac{z}{2 \pi}\right)-u_{n}(z)\right| d z \\
& =\int_{0}^{h} \sum_{\kappa=0}^{n}\left(B_{s}\left(\frac{z+\kappa h}{2 \pi}\right)-u_{n}(z+\kappa h)\right) \operatorname{sgn} B_{s}\left((n+1) \frac{z}{2 \pi}\right) d z .
\end{aligned}
$$

We apply now

$$
\sum_{\kappa=0}^{n} B_{s}\left(\frac{z+\kappa h}{2 \pi}\right)=(n+1)^{1-s} B_{s}\left((n+1) \frac{z}{2 \pi}\right)
$$


(the "multiplication theorem") and

$$
\sum_{\kappa=0}^{n} u_{n}(z+\kappa h)=0
$$

(an identity holding for all $u \in \mathscr{T}_{n}$ ) and get

$$
\begin{aligned}
\int_{0}^{2 \pi}\left|B_{s}\left(\frac{z}{2 \pi}\right)-u_{n}(z)\right| d z & =(n+1)^{1-s} \int_{0}^{h}\left|B_{s}\left((n+1) \frac{z}{2 \pi}\right)\right| d z \\
& =(n+1)^{-s} 2 \pi \int_{0}^{1}\left|B_{s}(x)\right| d x .
\end{aligned}
$$

Combining this relation with (3.3) gives assertion (ii) of the theorem.

\section{Proof of Theorem 6}

Lemma 2. Let $f \in \mathscr{K}_{s}$; then there exist $2 \pi$-periodic functions $h, j, t$ such that

$$
f(\cos y)=h(y) \sin ^{s} y+j(y)+t(y)
$$

with

(i) $\inf \left\{\left\|h^{(s)}-c\right\|: c \in \mathbb{R}\right\} \leq 1$,

(ii) $\left\|j^{(s+1)}\right\| \leq$ const $_{s}$, where const $s_{s}$ does not depend on $f$,

(iii) $t \in \mathscr{T}_{s}$.

Proof. We begin with Taylor's formula:

$$
f(x)=\sum_{\nu=0}^{s-1} \nu !^{-1} f^{(\nu)}(0) x^{\nu}+(s-1) !^{-1} \int_{0}^{x} f^{(s)}(u)(x-u)^{s-1} d u .
$$

If we put $x=\cos y$ and substitute $u=\cos v$, we get

$$
\begin{aligned}
f(\cos y)= & \sum_{\nu=0}^{s-1} \nu !^{-1} f^{(\nu)}(0) \cos ^{\nu} y-(s-1) !^{-1} \\
& \times \int_{\pi / 2}^{y} f^{(s)}(\cos v)(\cos y-\cos v)^{s-1} \sin v d v
\end{aligned}
$$

Using the notation

$$
g:=(\cos y-\cos (\cdot))^{s-1} \sin (\cdot) \quad \text { and } \quad h_{0}:=f^{(s)}(\cos \cdot)-\frac{1}{2} a_{0}\left[f^{(s)}(\cos \cdot)\right],
$$

we obtain

$$
\begin{aligned}
f(\cos y)= & \sum_{\nu=0}^{s-1} \nu !^{-1} f^{(\nu)}(0) \cos ^{\nu} y-\frac{1}{2} a_{0}\left[f^{(s)}(\cos \cdot)\right](s-1) !^{-1} \int_{\pi / 2}^{y} g(v) d v \\
& -(s-1) !^{-1} \int_{\pi / 2}^{y} h_{0}(v) g(v) d v \\
=: & \tilde{t}(y)-(s-1) !^{-1} \int_{\pi / 2}^{y} h_{0}(v) g(v) d v .
\end{aligned}
$$


We proceed with $s$ partial integrations and denote by $h_{1}, h_{2}, \ldots, h_{s}$ the successive primitives of $h_{0}$ with mean value zero. We obtain

$$
\begin{aligned}
\int_{\pi / 2}^{y} h_{0}(v) g(v) d v= & {\left[h_{1} g-h_{2} g^{\prime}+-\cdots+(-1)^{s-1} h_{s} g^{(s-1)}\right]_{\pi / 2}^{y} } \\
& +(-1)^{s} \int_{\pi / 2}^{y} h_{s}(v) g^{(s)}(v) d v .
\end{aligned}
$$

Using induction, we get easily

$$
\begin{aligned}
g^{(\kappa)}(v)= & (\cos y-\cos v)^{s-1-\kappa}(s-1)(s-2) \cdots(s-\kappa) \sin ^{\kappa+1} v \\
& +\frac{1}{2}(\cos y-\cos v)^{s-\kappa}(s-1)(s-2) \cdots(s-\kappa+1)(\kappa+1) \kappa \sin ^{\kappa-1} v \cos v \\
& +(\cos y-\cos v)^{s-\kappa+1} T_{\kappa, s}
\end{aligned}
$$

with $T_{\kappa, s} \in \mathscr{T}_{\kappa-1}$. Hence, we have immediately

$$
\begin{gathered}
g^{(\kappa)}(y)=0, \quad \kappa=0,1, \ldots, s-2, \\
g^{(s-1)}(y)=(s-1) ! \sin ^{s} y,
\end{gathered}
$$

and with a simple computation

$$
g^{(s)}(y)=\frac{1}{2}(s+1) ! \sin ^{s-1} y \cos y .
$$

From all this we get

$$
\begin{aligned}
\int_{\pi / 2}^{y} h_{0}(v) g(v) d v=\tilde{\tilde{t}}(y)+(-1)^{s-1}(s-1) ! h_{s}(y) \sin ^{s} y \\
\\
+(-1)^{s} \int_{\pi / 2}^{y} h_{s}(v) g^{(s)}(v) d v
\end{aligned}
$$

with $\tilde{\tilde{t}} \in \mathscr{T}_{s}$. If we substitute this equation in (4.2), we arrive at (4.1) with

$$
\begin{aligned}
t & =\tilde{t}-(s-1) !^{-1} \tilde{\tilde{t}}, \quad h=(-1)^{s} h_{s}, \\
j(y) & =(-1)^{s+1}(s-1) !^{-1} \int_{\pi / 2}^{y} h_{s}(v) g^{(s)}(v) d v,
\end{aligned}
$$

and assertions (i) and (iii) are evident. In view of

$$
j^{\prime}(y)=(-1)^{s+1}(s-1) !^{-1} h_{s}(y) g^{(s)}(y)=(-1)^{s+1} \frac{1}{2} s(s+1) h_{s}(y) \sin ^{s-1} y \cos y
$$

$j^{(s+1)}$ exists, and we have $\left\|j^{s+1}\right\| \leq$ const $\cdot \max \left\{\left\|h_{0}\right\|, \ldots,\left\|h_{s}\right\|\right\}$. But observe that $h_{\kappa}$ has mean value zero; therefore, $\left\|h_{\kappa}\right\| \leq 2 \pi\left\|h_{\kappa-1}\right\|$ and $\left\|j^{(s+1)}\right\| \leq$ const $_{1}\left\|h_{0}\right\| \leq 2$ const $_{1}$. This is assertion (ii).

Proof of Theorem 6. We represent $f(\cos \cdot)$ as in (4.1). We approximate $h$ by $t_{n-s} \in \mathscr{T}_{n-s}$ according to Theorem 7. The function $j$ in (4.1) can be approximated by $\hat{t}_{n} \in \mathscr{T}_{n}$ according to Jackson's theorem, so that we have

$$
\left\|j-\hat{t}_{n}\right\| \leq \mathrm{const} n^{-s-1} \text {. }
$$

Let

$$
\tilde{p}_{n}(y):=t_{n-s}(y) \sin ^{s} y+\hat{t}_{n}(y)+t(y) .
$$


We have

$$
\begin{aligned}
|f(\cos y)-\tilde{p}(y)| & \leq\left|h(y)-t_{n-s}(y)\right| \sin ^{s} y+\left|j(y)-\hat{t}_{n}(y)\right| \\
& \leq\left(\frac{2 \pi}{n+1-s}\right)^{s}\left|\sin ^{s} y\right| \int_{0}^{1}\left|B_{s}(u)\right| d u+\text { const } n^{-s-1} .
\end{aligned}
$$

Here, $\tilde{p}$ is an even function (if not, replace it by its even part); therefore the substitution $y=\arccos x$ leads immediately $\left(p_{n}:=\tilde{p}_{n}(\arccos \cdot)\right)$ to assertions (i) and (ii) of Theorem 6.

The proof of assertion (iii) starts with

$$
\hat{a}_{\nu}\left[f-p_{n}\right]=a_{\nu}\left[f(\cos \cdot)-\tilde{p}_{n}\right]=a_{\nu}\left[\left(h-t_{n-s}\right) \sin ^{s} \cdot\right]+a_{\nu}\left[j-\hat{t}_{n}\right] .
$$

We have the identity

$$
a_{\nu}\left[g \sin ^{s} \cdot\right]=\sum_{\lambda=\nu-s}^{\nu+s}\left(\alpha_{\lambda} a_{|\lambda|}[g]+\beta_{\lambda} b_{|\lambda|}[g]\right),
$$

where $\alpha_{\lambda}, \beta_{\lambda}$ denote some constants. Using this identity and assertion (iii) of Theorem 7, we obtain

$$
a_{\nu}\left[\left(h-t_{n-s}\right) \sin ^{s} \cdot\right]=O\left(n^{-s-1}\right),
$$

and this gives the bound for the first term in (4.4). The second term can (using (4.3)) be bounded by $\left\|a_{\nu}\right\|\left\|j-\hat{t}_{n}\right\| \leq 2$ const $n^{-s-1}$.

\section{Proof of Theorem 1}

Let $f \in \mathscr{K}_{s}$, and choose $p_{m}$ according to Theorem 6. Then we have

$$
\begin{aligned}
\left|I[f]-Q_{m}[f]\right|= & \left|I\left[f-p_{m}\right]-Q_{m}\left[f-p_{m}\right]\right| \\
\leq & \left|I\left[f-p_{m}\right]\right|+Q_{m}\left[\left|f-p_{m}\right|\right] \\
\leq & \left|I\left[f-p_{m}\right]\right|+\left(\frac{2 \pi}{m+1}\right)^{s} Q_{m}\left[h^{s}\right] \int_{0}^{1}\left|B_{s}(u)\right| d u \\
& +\left\|Q_{m}\right\| O\left(m^{-s-1}\right)
\end{aligned}
$$

with $h(x)=\sqrt{1-x^{2}}$. We now use $\left\|Q_{m}\right\|=Q_{m}[1]=I[1]=\|I\|$ and $Q_{m}[f] \rightarrow$ $I[f]$ (this follows from $\operatorname{deg}\left[Q_{m}\right] \rightarrow \infty$ ) and obtain

$$
\left|I[f]-Q_{m}[f]\right| \leq\left(\frac{2 \pi}{m+1}\right)^{s} I\left[h^{s}\right] \int_{0}^{1}\left|B_{s}(u)\right| d u+o\left(m^{-s}\right)+\left|I\left[f-p_{m}\right]\right| .
$$

It is now only necessary to prove that

$$
\lim _{m \rightarrow \infty} m^{s}\left|I\left[f-p_{m}\right]\right|=0
$$

holds uniformly in $\mathscr{K}_{s}$.

For every positive $\varepsilon$ there exist numbers $\gamma_{1}, \ldots, \gamma_{r}$ such that

$$
\int_{0}^{\pi}\left|k(\cos t) \sin t-\frac{2}{\pi} \sum_{\nu=0}^{r} \gamma_{\nu} \cos \nu t\right| d t<\varepsilon .
$$

If we substitute $x=\cos t$, the same formula reads

$$
\int_{-1}^{1}\left|k(x)-\frac{2}{\pi} \sum_{\nu=0}^{r} \gamma_{\nu}\left(1-x^{2}\right)^{-1 / 2} T_{\nu}(x)\right| d x<\varepsilon
$$


This means that the functional

$$
I^{*}[f]:=\sum_{\nu=0}^{r} \gamma_{\nu} \hat{a}_{\nu}[f]
$$

approximates the given functional $I$; more precisely, $\left|I[f]-I^{*}[f]\right| \leq \varepsilon\|f\|$. We have

$$
\begin{aligned}
\left|I\left[f-p_{m}\right]\right| & \leq\left|\left(I-I^{*}\right)\left[f-p_{m}\right]\right|+\left|I^{*}\left[f-p_{m}\right]\right| \\
& \leq \varepsilon\left\|f-p_{m}\right\|+\left|\sum_{\nu=0}^{r} \gamma_{\nu} \hat{a}_{\nu}\left[f-p_{m}\right]\right|,
\end{aligned}
$$

and if we apply Theorem 6 (ii) and (iii), then we have proved (5.1).

\section{Proof of Theorem 2}

Lemma 3. Let $a \leq x_{1}<x_{2}<\cdots<x_{n} \leq b$. Then there exists

$$
\begin{gathered}
g \in \mathscr{K}_{s}[a, b] \quad \text { with } g\left(x_{\nu}\right)=0(\nu=1, \ldots, n), \\
g^{(\lambda)}(a)=g^{(\lambda)}(b)=0 \quad(\lambda=0,1, \ldots, s-1)
\end{gathered}
$$

and

$$
\int_{a}^{b} g(x) d x \geq \frac{(b-a)^{s+1} K_{s}}{(2 \pi)^{s}(n+2 s)^{s}} .
$$

Proof. Motornyi [9] established the existence of a function $g \in \mathscr{K}_{s}[0,2 \pi]$ such that we have $g\left(x_{\nu}\right)=0$ for given points $0 \leq x_{1}<\cdots<x_{n}<2 \pi$ and

$$
\int_{0}^{2 \pi} g(x) d x \geq \frac{2 \pi K_{s}}{n^{s}} .
$$

We apply this theorem to a situation where we have added $s$ further points $y_{1}, \ldots, y_{s}$ near 0 and $s$ further points $z_{1}, \ldots, z_{s}$ near $2 \pi$ to the fixed points $x_{1}, \ldots, x_{n}$. If we now let $y_{\nu} \rightarrow 0$ and $z_{\nu} \rightarrow \pi(\nu=1, \ldots, s)$ and use compactness arguments, we obtain the existence of a function $g \in \mathscr{K}_{s}[0,2 \pi]$ with $g\left(x_{\nu}\right)=g^{(\lambda)}(0)=g^{(\lambda)}(2 \pi)=0(\nu=1, \ldots, n ; \lambda=0, \ldots, s-1)$ and

$$
\int_{0}^{2 \pi} g(x) d x \geq \frac{2 \pi K_{s}}{(n+2 s)^{s}} .
$$

The lemma now follows by a simple transformation.

Proof of Theorem 2. Fix $\varepsilon>0$. There exists a step function $t \geq 0$ with

$$
\int_{-1}^{1}|k(x)-t(x)| d x<\varepsilon .
$$

Denote by $-1=\xi_{0}<\xi_{1}<\cdots<\xi_{m}=1$ a partition of $[-1,1]$ such that all Riemann sums of the integral

$$
J:=\int_{-1}^{1} t(x)(v(x))^{-s} d x
$$

which are based on this partition are contained in the interval $[J-\varepsilon, J+\varepsilon]$. There are partitions of this type; we may even add the further hypothesis that the knots of $t$ are a subset of $\left\{\xi_{0}, \ldots, \xi_{m}\right\}$. 
If $g_{n} \in \mathscr{K}_{s}$ has the zeros $x_{\nu, n}(\nu=1, \ldots, n)$, then we have

$$
\begin{aligned}
\rho_{s}\left(Q_{n}\right) & \geq I\left[g_{n}\right] \geq \int_{-1}^{1} g_{n}(x) t(x) d x-\varepsilon\left\|g_{n}\right\| \\
& =\sum_{\nu=0}^{m-1} t\left(\xi_{\nu}+0\right) \int_{\xi_{\nu}}^{\xi_{\nu+1}} g_{n}(x) d x-\varepsilon\left\|g_{n}\right\| .
\end{aligned}
$$

We construct a function $g_{n}$ piecewise on each of the intervals $\left[\xi_{\mu}, \xi_{\mu+1}\right]$ by an application of Lemma 3 . The points $x_{1}, \ldots, x_{n}$ of the lemma consist of the points $x_{\nu, n}$ situated in $\left[\xi_{\mu}, \xi_{\mu+1}\right]$ together with some added points, which guarantee that the distance between adjacent points is $\leq d n^{-1}$, where $d$ is a positive constant. This can be done with $\left\lceil n\left(\xi_{\mu+1}-\xi_{\mu}\right) / d\right\rceil+1$ extra points; therefore, we have not more than

$$
\begin{aligned}
& \left\lceil n \frac{\xi_{\mu+1}-\xi_{\mu}}{d}\right\rceil+1+n \int_{\xi_{\mu}}^{\xi_{\mu+1}} v(x) d x(1+o(1)) \\
& \quad=n\left(\xi_{\mu+1}-\xi_{\mu}\right)\left(d^{-1}+v\left(\eta_{\nu}\right)\right)(1+o(1)) \\
& \quad \leq n\left(\xi_{\mu+1}-\xi_{\mu}\right) v\left(\eta_{\nu}\right)\left(1+d^{-1} M^{-1}\right)(1+o(1)) \quad\left(\eta_{\nu} \in\left[\xi_{\mu}, \xi_{\mu+1}\right]\right)
\end{aligned}
$$

prescribed zeros in $\left[\xi_{\mu}, \xi_{\mu+1}\right]$. From Lemma 3 there now follows the existence of $g_{\mu, n} \in \mathscr{K}_{s}\left[\xi_{\mu}, \xi_{\mu+1}\right]$ with

$$
\int_{\xi_{\mu}}^{\xi_{\mu+1}} g_{\mu, n}(x) d x \geq \frac{K_{s}}{(2 \pi)^{s}\left(1+d^{-1} M^{-1}\right)^{s} n^{s}} \frac{\xi_{\mu+1}-\xi_{\mu}}{\left(v\left(\eta_{\mu}\right)\right)^{s}}(1+o(1)) .
$$

We have shown: If $n>n_{0}(\varepsilon)$, then

$$
\begin{aligned}
\int_{\xi_{\mu}}^{\xi_{\mu+1}} g_{\mu, n}(x) d x \geq(1-\varepsilon) \frac{K_{s}}{(2 \pi)^{s}\left(1+d^{-1} M^{-1}\right)^{s} n^{s}} \frac{\xi_{\mu+1}-\xi_{\mu}}{\left(v\left(\eta_{\mu}\right)\right)^{s}} & \\
\mu & =0,1, \ldots, m-1 .
\end{aligned}
$$

We put $g_{n}(x):=g_{\mu, n}(x)$ if $x \in\left[\xi_{\mu}, \xi_{\mu+1}\right]$ and obtain $g_{n} \in \mathscr{K}_{s}$. In view of the definition of the $\xi_{\mu}$ we have

$$
\begin{aligned}
& \sum_{\mu=0}^{m-1} t\left(\xi_{\mu}+0\right)+\int_{\xi_{\mu}}^{\xi_{\mu+1}} g_{n}(x) d x \\
& \quad \geq(1-\varepsilon) \frac{K_{s}}{(2 \pi)^{s}\left(1+d^{-1} M^{-1}\right)^{s} n^{s}}\left(\int_{-1}^{1} \frac{t(x)}{(v(x))^{s}} d x-\varepsilon\right),
\end{aligned}
$$

and by virtue of (6.1) we arrive at

$$
\begin{aligned}
& \sum_{\mu=0}^{m-1} t\left(\xi_{\mu}+0\right) \int_{\xi_{\mu}}^{\xi_{\mu+1}} g_{n}(x) d x \\
& \quad \geq(1-\varepsilon) \frac{K_{s}}{(2 \pi)^{s}\left(1+d^{-1} M^{-1}\right)^{s} n^{s}}\left(I\left[v^{-s}\right]-M^{-s} \varepsilon-\varepsilon\right) .
\end{aligned}
$$

Let $x$ be fixed. Then there are zeros $y_{1}, \ldots, y_{s}$ of $g_{n}$ with

$$
\sup \left\{\left|x-y_{\nu}\right|: \nu=1, \ldots, s\right\} \leq \frac{s d}{n} \text {. }
$$


Since $g_{n} \in \mathscr{K}_{s}$, we have $\left|\operatorname{dvd}\left(x, y_{1}, \ldots, y_{s}\right)\left[g_{n}\right]\right| \leq \frac{1}{s !}$, where $\operatorname{dvd}(\cdots)\left[g_{n}\right]$ means the divided difference of $g_{n}$ for the indicated points. The well-known expression of $\mathrm{dvd}$ as a linear combination of $g_{n}(x)$ and $g_{n}\left(y_{\nu}\right)$ leads to

$$
\left|g_{n}(x)\right| \leq \frac{1}{s !} \prod_{\nu=1}^{s}\left|x-y_{\nu}\right| \leq \frac{1}{s !}\left(\frac{s d}{n}\right)^{s}
$$

and thus we have

$$
\left\|g_{n}\right\| \leq \frac{1}{s 1}\left(\frac{s d}{n}\right)^{s} .
$$

We substitute (6.3) and (6.4) in (6.2) and get

$$
\rho_{s}\left(Q_{n}\right) \geq(1-\varepsilon) \frac{K_{s}}{(2 \pi)^{s}\left(1+d^{-1} M^{-1}\right)^{s} n^{s}}\left(I\left[v^{-s}\right]-M^{-s} \varepsilon-\varepsilon\right)-\varepsilon \frac{1}{s !}\left(\frac{s d}{n}\right)^{s}
$$

if $n>n_{0}(\varepsilon)$. This implies

$\varliminf_{n \rightarrow \infty} n^{s} \rho_{s}\left(Q_{n}\right) \geq(1-\varepsilon) \frac{K_{s}}{(2 \pi)^{s}\left(1+d^{-1} M^{-1}\right)^{s}}\left(I\left[v^{-s}\right]-M^{-s} \varepsilon-\varepsilon\right)-\varepsilon \frac{1}{s !}\left(\frac{s d}{n}\right)^{s}$.

This relation holds for any $\varepsilon$ and for any $d$; therefore, the assertion of the theorem follows.

\section{Proof of Theorem 5}

Our starting point is the expansion in Chebyshev polynomials

$$
f=\frac{1}{2} \hat{a}_{0}[f]+\sum_{\nu=1}^{\infty} \hat{a}_{\nu}[f] T_{\nu} .
$$

This expansion is uniformly convergent if $f$ has a bounded derivative, that is, in all cases of interest in this paper.

If $Q$ satisfies $\operatorname{deg}[Q] \geq m$, then we have

$$
R[f]=\hat{a}_{m+1}[f] R\left[T_{m+1}\right]+R\left[\sum_{\nu>m+1} \hat{a}_{\nu}[f] T_{\nu}\right]
$$

hence

$$
\begin{aligned}
& \left|\hat{a}_{m+1}[f] R\left[T_{m+1}\right]\right|-\|R\|\left\|\sum_{\nu>m+1} \hat{a}_{\nu}[f] T_{\nu}\right\| \\
& \quad \leq|R[f]| \leq\left|\hat{a}_{m+1}[f] R\left[T_{m+1}\right]\right|+\|R\|\left\|\sum_{\nu>m+1} \hat{a}_{\nu}[f] T_{\nu}\right\|,
\end{aligned}
$$

and thus, using $\|R\| \leq 2\|I\|$,

$$
\begin{aligned}
& \sup \left\{\left|\hat{a}_{m+1}[f]\right|: f \in \mathscr{K}_{m-l+1}\right\} \sup \left\{\left|R\left[T_{m+1}\right]\right|: \operatorname{deg}[Q] \geq m\right\} \\
& -2\|I\| \sup \left\{\left\|\sum_{\nu>m+1} \hat{a}_{\nu}[f] T_{\nu}\right\|: f \in \mathscr{K}_{m-l+1}\right\} \\
& \leq \rho_{m-l+1, m} \leq \cdots+\cdots
\end{aligned}
$$


We need the following results from [2]:

$$
\begin{aligned}
& \sup \left\{\left|\hat{a}_{m+1}[f]\right|: f \in \mathscr{K}_{m-l+1}\right\} \\
& \quad=c_{l} \frac{m^{1 / 2}}{\sqrt{\pi} 2^{m}(m+1) !}(1+o(1)) \quad(m \rightarrow \infty, \text { fixed }), \\
& \sup \left\{\left\|\sum_{\nu>m+1} \hat{a}_{\nu}[f] T_{\nu}\right\|: f \in \mathscr{K}_{m-l+1}\right\} \\
&=c_{l+1} \frac{m^{(l+1) / 2}}{\sqrt{\pi} 2^{m+1}(m+1) !}(1+o(1)) \quad(m \rightarrow \infty, l \text { fixed }) .
\end{aligned}
$$

For the estimation of the remaining term in (7.1) we use the following theorem of Markoff and Krein (see, e.g., [6, Theorem 6]): If $\operatorname{deg}[Q] \geq m$ and $f^{(m+1)} \geq 0$, then

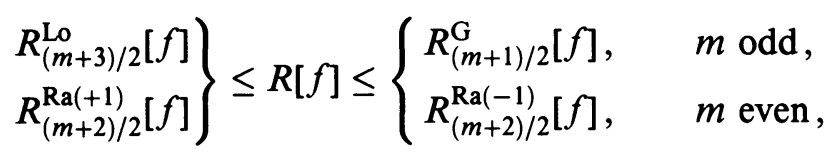

where $R^{\mathrm{G}}, R^{\mathrm{Lo}}, R^{\mathrm{Ra}(+1)}, R^{\mathrm{Ra}(-1)}$ denote the errors of the quadrature rules of Gauss, Lobatto, Radau with abscissa +1 , Radau with abscissa -1 , and the indicated number of evaluation points.

We apply this theorem to $f=T_{m+1}$ and obtain

$$
\left|R\left[T_{m+1}\right]\right| \leq\left|R^{\mathrm{spec}}\left[T_{m+1}\right]\right|
$$

where $R^{\text {spec }}$ is the error of one of the specified quadrature rules. We assume $R^{\text {spec }}=R_{(m+3) / 2}^{\mathrm{Lo}}$; in the other cases the proof is similar.

Denote by $q$ the orthonormal polynomial of degree $\frac{m-1}{2}$ associated with the weight function $k^{*}(x):=\left(1-x^{2}\right) k(x)$, and denote by $\delta_{m}$ its leading coefficient. The abscissas of $Q_{(m+3) / 2}^{\mathrm{Lo}}$ are the zeros of $q^{*}(x):=\left(x^{2}-1\right) q(x)$. Hence we have

$$
R_{(m+3) / 2}^{\mathrm{Lo}}\left[T_{m+1}\right]=2^{m} \delta_{m}^{-2} R_{(m+3) / 2}^{\mathrm{Lo}}\left[q^{*} q\right]=2^{m} \delta_{m}^{-2} I\left[q^{*} q\right]=-2^{m} \delta_{m}^{-2} .
$$

Szegö ([11, p. 302]) proved

$$
\begin{aligned}
\delta_{m}=\pi^{-1 / 2} 2^{(m-1) / 2} \exp \left(-\frac{1}{2 \pi} \int_{-1}^{1} \frac{\ln k(x)\left(1-x^{2}\right)}{\sqrt{1-x^{2}}} d x\right)(1+o(1)) & \\
& (m \rightarrow \infty) .
\end{aligned}
$$

Using

$$
\int_{-1}^{1} \frac{\ln \left(1-x^{2}\right)}{\sqrt{1-x^{2}}} d x=2 \int_{0}^{\pi} \ln \sin t d t=-2 \pi \ln 2,
$$

we can write (7.5) as

$$
\delta_{m}=\pi^{-1 / 2} 2^{(m+1) / 2} \exp \left(-\frac{1}{2 \pi} \int_{-1}^{1} \frac{\ln k(x)}{\sqrt{1-x^{2}}} d x\right)(1+o(1)) .
$$

Combining this with (7.4) gives

$$
\left|R_{(m+3) / 2}^{\mathrm{Lo}}\left[T_{m+1}\right]\right|=\frac{\pi}{2} \exp \left(\frac{1}{\pi} \int_{-1}^{1} \frac{\ln k(x)}{\sqrt{1-x^{2}}} d x\right)(1+o(1))
$$


we arrive at the same result, if $R^{\text {spec }} \in\left\{R_{(m+1) / 2}^{\mathrm{G}}, R_{(m+2) / 2}^{\mathrm{Ra}(+1)}, R_{(m+2) / 2}^{\mathrm{Ra}(-1)}\right\}$. Therefore, we have

(7.6) $\sup \left\{\left|R\left[T_{m+1}\right]\right|: \operatorname{deg}(Q) \geq m\right\}=\frac{\pi}{2} \exp \left(\frac{1}{\pi} \int_{-1}^{1} \frac{\ln k(x)}{\sqrt{1-x^{2}}} d x\right)(1+o(1))$.

Substitution of (7.2), (7.3), and (7.6) in (7.1) gives us immediately the asymptotics of $\rho_{m-l+m, m}$, and the proof shows that the bound for the error is attained for the Gaussian rule.

\section{BIBLIOGRAPHY}

1. H. Brass, Quadraturverfahren, Vandenhoeck und Ruprecht, Göttingen, 1977.

2. __ Restabschätzungen zur Polynomapproximation, Numerische Methoden der Approximationstheorie Bd. 7 (L. Collatz, G. Meinardus, and H. Werner, eds.), Birkhäuser Verlag, Basel, 1984.

3. __ Eine Fehlerabschätzung für positive Quadraturformeln, Numer. Math. 47 (1985), 395399.

4. __ Error bounds based on approximation theory, Numerical Integration-Recent Developments, Software and Applications (T. D. Espelid and A. Genz, eds.), NATO ASI Series, Series C: Mathematical and Physical Sciences, vol. 357, Kluwer, Dordrecht, 1992, pp. 147163.

5. H. Brass and K.-J. Förster, Error bounds for quadrature formulas near Gaussian quadrature, J. Comput. Appl. Math. 28 (1989), 145-154.

6. H. Brass and G. Schmeisser, Error estimates for interpolatory quadrature formulae, Numer. Math. 37 (1981), 371-386.

7. G. Freud, Orthogonal polynomials, Pergamon Press, New York, 1971.

8. G. Hämmerlin and K.-H. Hoffmann, Numerical mathematics, Springer-Verlag, New York, 1991.

9. V. P. Motornyi, On the best quadrature formula of the form $\sum_{k=1}^{n} p_{k} f\left(x_{k}\right)$ for some classes of differentiable periodic functions, Math. USSR Izv. 8 (1974), 591-620.

10. K. Petras, Asymptotic behaviour of Peanokernels of fixed order, Numerical Integration III (H. Braß and G. Hämmerlin, eds.), Birkhäuser Verlag, Basel, 1988, pp. 186-198.

11. G. Szegö, Orthogonal polynomials, Amer. Math. Soc. Colloq. Publ., vol. 23, 4th ed., Amer. Math. Soc., Providence, RI, 1975.

12. A. F. Timan, Theory of approximation of functions of a real variable, Macmillan, New York, 1963.

Institut für Angewandte Mathematik, Technische Universität Braunschweig, Pockelsstr. 14, W-3300 BraunschWeig, Germany 